\title{
Frailty status in older adults is related to alterations in indoleamine 2,3-dioxygenase 1 and guanosine triphosphate cyclohydrolase I enzymatic pathways
}

\author{
Diego Marcos-Pérez MSc ${ }^{\text {ab }}$, María Sánchez-Flores MSc ${ }^{\mathrm{ab}}$, Ana Maseda $\mathrm{PhD}^{\mathrm{c}}$, \\ Laura Lorenzo-López $\mathrm{PhD}^{\mathrm{c}}$, José C. Millán-Calenti $\mathrm{PhD}^{\mathrm{c}}$, Barbara Strasser $\mathrm{PhDd}$, \\ Johanna M. Gostner PhD ${ }^{\mathrm{d}}$, Dietmar Fuchs $\mathrm{PhD}^{\mathrm{d}}$, Eduardo Pásaro $\mathrm{PhD}^{\mathrm{a}}$, Vanessa \\ Valdiglesias $\mathrm{PhD}^{\mathrm{a}}$, Blanca Laffon $\mathrm{PhD}^{\mathrm{a}}$
}

\author{
${ }^{a}$ DICOMOSA Group, Department of Psychology, Area of Psychobiology, Universidade da Coruña, A \\ Coruña, Spain \\ ${ }^{b}$ Department of Cell and Molecular Biology, Universidade da Coruña, A Coruña, Spain \\ ${ }^{c}$ Gerontology Research Group, Universidade da Coruña, Instituto de Investigación Biomédica de A Coruña \\ (INIBIC), Complexo Hospitalario Universitario de A Coruña (CHUAC), SERGAS, A Coruña, Spain \\ ${ }^{d}$ Biocenter, Innsbruck Medical University, Innsbruck, Austria
}

\begin{abstract}
Background. Frailty is a multidimensional syndrome correlated to the loss of homeostasis and increased vulnerability to stressors, which is associated with increase in the risk of disability, comorbidity, hospitalization, and death in the elderly. It is based on the interplay of physiological, psychological, social, and environmental factors.

Objectives. Because aging involves a detrimental immune response, this work aimed to assess the possible role of chronic low-grade immune stimulation on frailty status in the elderly.

Methods. Biomarkers involved in indoleamine 2,3-dioxygenase 1 and guanosine triphosphate cyclohydrolase I enzymatic pathways (namely neopterin, tryptophan, kynurenine, phenylalanine, tyrosine, and nitrite) were analyzed in a population of Spanish older adults aged 65 years and above, and their relationships with frailty status were evaluated.

Results. Significant increases in neopterin levels, kynurenine/tryptophan ratio, and phenylalanine/tyrosine ratio, and significant decreases in tryptophan, nitrite and tyrosine concentrations in frail individuals compared with nonfrail persons were obtained. Significant correlations were also observed between immune biomarkers, indicating they change in parallel, thus, pointing to interrelated causes. Besides, reference ranges for a number of immune biomarkers in the population of robust older adults were established for the first time.

Conclusions. Results obtained in the present study are consistent with the idea that frailty status in the elderly is associated with an additional degree of immune stimulation, manifested in a more intense disturbance of indoleamine 2,3-dioxygenase 1 and guanosine triphosphate cyclohydrolase I pathways than in nonfrail or prefrail older adults.
\end{abstract}

Keywords

Frailty; Immune activation; Kynurenine/tryptophan ratio neopterin; Nitrite; Phenylalanine/tyrosine ratio 
Average age of populations around the world is rapidly increasing, and this trend is evident from the most developed countries to the lowest income regions. ${ }^{1}$ In Europe, by 2060, those aged $65 \pm$ years will comprise $30 \%$ of the population, and 1 person in 8 will be aged 80 years or more. ${ }^{2}$ This aging situation leads to an increase in future healthcare expenditure. Because of that, researchers and governments are interested in increasing our knowledge about aging and agerelated conditions and disorders, to reduce sanitary and socioeconomic costs in the future.

Chronological age is normally used to classify elderly people; however, because of the great heterogeneity reported in the aging process, chronological age is not a good indicator of aging signs and symptoms. In this regard, the term "frailty" represents an approach to age-related conditions by replacing the obsolete concept of "chronological age" with the more accurate and person-tailored parameter of "biological age."3 Frailty has been defined as a medical syndrome with multiple causes and contributors that is characterized by diminished strength, endurance, and reduced physiologic function that increases an individual's vulnerability for developing increased dependency and/or death. ${ }^{4}$ It may be initiated by disease, lack of activity, inadequate nutritional intake, stress, and/or the physiologic changes of aging. It is manifested as loss of skeletal muscle mass (sarcopenia), abnormal function of immune and neuroendocrine systems, and poor energy regulation. ${ }^{5}$ Causes of frailty are complex; it is a multidimensional syndrome based on the interplay of genetic, biological (hormonal, metabolic, and immune-inflammatory systems), physical, psychological, social, and environmental factors. ${ }^{6,7}$ Prevalence of frailty is highly variable and dependent on a number of factors including features of the population evaluated and frailty scale applied. This prevalence has been estimated to range from $4 \%$ to $59.1 \%{ }^{8}$ In community-dwelling Spanish populations, it was established to be $8.6 \%{ }^{9}$ and $16.3 \%{ }^{10}$ in different studies employing Fried's criteria ${ }^{11}$ for frailty identification. However, according to a recent crosssectional study with 331 Spanish participants of both sexes, this prevalence can reach $68.8 \%$ in the case of institutionalized older people. ${ }^{12}$

Normally in later life, immune response becomes chronic and detrimental, contributing to the development of a number of agerelated chronic diseases such as atherosclerosis, ${ }^{13}$ type-2 diabetes, ${ }^{14}$ Alzheimer disease, ${ }^{15}$ and osteoporosis. ${ }^{16}$ Thus, the term "immunosenescence" reflects those age-related changes in immune responses, both cellular and serologic, affecting the process of generating specific responses to foreign and self-antigens. ${ }^{17}$ Although inflammation is an acute and fundamental response to cope with internal and external damaging agents, the continuous antigenic stress means the immune system can become overstimulated over time and inefficient with age; this process has been proposed to be named "inflammaging." 18

Upon immune activation, inflammatory factors (eg, Th1-type cytokine interferon-g) induce the expression of the enzymes indoleamine 2,3-dioxygenase 1 (IDO) (EC 1.13.11.52) and guanosine triphosphate cyclohydrolase I (GCH) (EC 3.5.4.16) in monocytes/macrophages and dendritic cells (Figure 1). IDO is involved in transforming tryptophan into kynurenine. In vivo, kynurenine/tryptophan (Kyn/Trp) ratio reflects tryptophan breakdown, and in inflammatory conditions is considered to represent IDO enzyme activity. ${ }^{19}$ Several clinical conditions associated with increased immune activation are characterized by intensified tryptophan degradation [eg, several infections including human immunodeficiency virus (HIV) infection, autoimmune syndromes, a number of cancers, neurodegenerative disorders, and cardiovascular disease, among others]. ${ }^{20}$ When $\mathrm{GCH}$, the key enzyme of pteridine biosynthesis, is activated, it produces 7,8dihydroneopterin triphosphate $\left(\mathrm{NH}_{2} \mathrm{TP}\right)$, which is a precursor of neopterin and tetrahydrobiopterin $\left(\mathrm{BH}_{4}\right) . \mathrm{BH}_{4}$ is an essential cofactor of amino acid monooxygenases, including phenylalanine 4 hydroxylase (PHA) (EC 1.14.13.39), involved in the conversion of phenylalanine to tyrosine, and nitric oxide synthases (NOS) (EC 1.14.13.39), which catalyze the conversion of arginine to nitric oxide (NO). ${ }^{21}$ In conditions of immune activation, neopterin is released by activated human monocytic cells at the expense of the formation of $\mathrm{BH}_{4}{ }^{22}$ Thus, neopterin concentration in body fluids, including serum, urine, and cerebrospinal fluid, is considered a sensitive marker of immune system activation. In fact, neopterin levels are increased in malignant tumors, in autoimmune, cardiovascular, infectious, and neurodegenerative diseases, and during rejection episodes in allograft recipients. ${ }^{23}$ Likewise, the spectrum of diseases in which elevated serum phenylalanine levels, as consequence of low PHA activity, have been reported, including sepsis, HIV infection, cancer, burns, and trauma, ${ }^{24}$ is very similar to the one with increased degradation of tryptophan and neopterin production. 


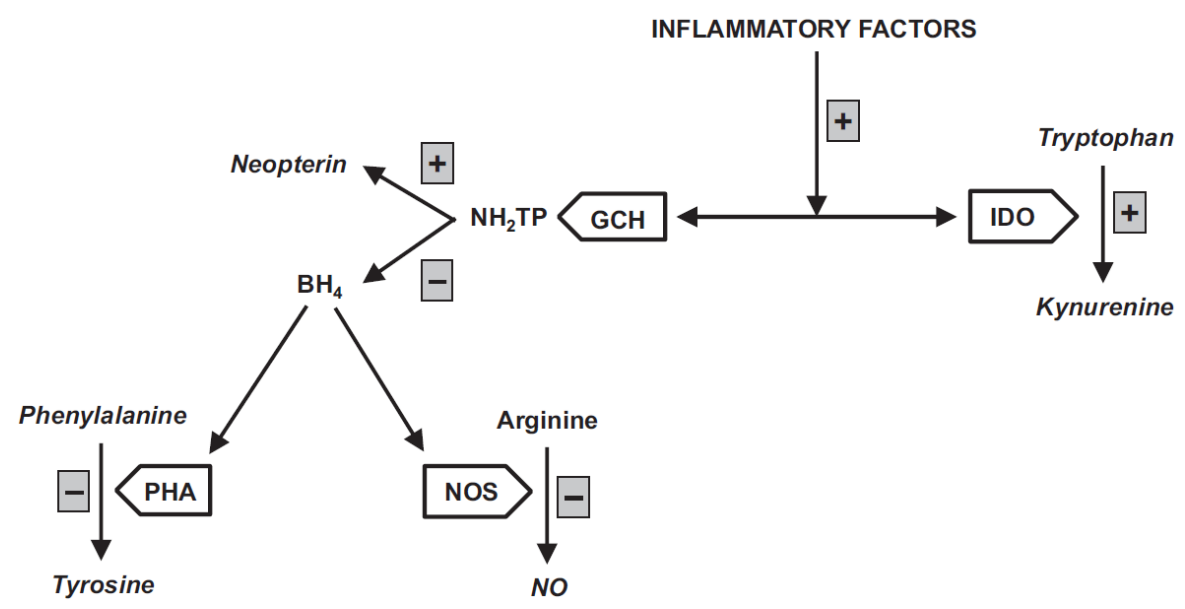

Fig. 1. Immune stimulation through inflammation factors involves activation of IDO and GCH pathways, which leads to increase in tryptophan breakdown, increase in neopterin production from 7,8-dihydroneopterin triphosphate $\left(\mathrm{NH}_{2} \mathrm{TP}\right)$ at the expense of $\mathrm{BH}_{4}$ and, consequently, decrease in PHA and NOS activities, resulting in decline of tyrosine and NO production. Italic letter indicates immune biomarkers analyzed in this study.

The present work aimed to assess the possible role of chronic low-grade immune stimulation on frailty status in the elderly. Our hypothesis was that immune stimulation biomarkers involved in IDO and GCH enzymatic pathways would have a significant association with frailty. To test this hypothesis, we conducted a cross-sectional study in a population of Spanish older adults analyzing neopterin, tryptophan, and phenylalanine metabolism parameters, and nitrite as an estimate of NO production, and evaluated their relationship with frailty status. In addition, we explored the potential relationship between the immune biomarkers and nutritional and functional status in older adults.

\section{Methods}

\section{Study Population}

Participants were recruited from several associations of retired or older people, nursing homes, and daycare centers located in Galicia, North-western Spain. The final cohort included 259 individuals ( 85 male and 174 female) aged 65 years and above. A clinical evaluation was carried out by trained interviewers to unify criteria, and participants completed a questionnaire to collect medical, lifestyle, and demographic information. In addition, whole blood samples were collected into vacutainer tubes without anticoagulant or containing sodium heparin between 9:30 AM and 12:30 PM, and were transported to the laboratory immediately. Serum and plasma samples were obtained by centrifugation at $2300 \mathrm{rpm}$ for 10 minutes, aliquoted, and stored at $-80^{\circ} \mathrm{C}$ until analysis. All laboratory measurements were performed in a blinded manner because of all samples were coded at the moment of collection. Exclusion criteria included taking medications known to affect the immune system and having autoimmune diseases, neoplasia, or any chronic infection, such as HIV, hepatitis C virus, and hepatitis B virus.

The general characteristics of the study population are collected in Table 1 . The number of current smokers and ex-smokers was quite low $(\mathrm{N}=5$ and $\mathrm{N}=49$, respectively); hence, they were combined in the category "ever smokers." Similarly, participants with low comorbidity (Charlson comorbidity index total score $=2, \mathrm{~N}=47$ ) and high comorbidity (total score $\geq 3, \mathrm{~N}=33$ ) ${ }^{25}$ were grouped together. And malnourished individuals $(\mathrm{N}=14)$ were combined with those at risk of malnutrition $(\mathrm{N}=80)$ in a single category. 
Table 1. Description of the Study Population

\begin{tabular}{|c|c|c|c|c|}
\hline Characteristic & Nonfrail & Prefrail & Frail & $P$ \\
\hline Total individuals $\mathrm{N}(\%)$ & $40(15.4)$ & $131(50.6)$ & $88(34.0)$ & \\
\hline Age $(y)^{*}$ & $73.2 \pm 5.5(65-85)$ & $77.05 \pm 7.7(65-100)$ & $85.8 \pm 7.9(65-102)$ & $<.001^{\dagger}$ \\
\hline \multicolumn{5}{|l|}{$\operatorname{Sex} N(\%)$} \\
\hline Male & $27(67.5)$ & $36(27.5)$ & $22(25.0)$ & \multirow[t]{2}{*}{$<.001$} \\
\hline Female & $13(32.5)$ & $95(72.5)$ & $66(75.0)$ & \\
\hline \multicolumn{5}{|l|}{ Living conditions $\mathrm{N}(\%)$} \\
\hline Family home & $40(100)$ & $113(86.3)$ & $5(5.7)$ & \multirow[t]{3}{*}{$<.001$} \\
\hline Family home + daycare center & - & $4(3.1)$ & $23(26.1)$ & \\
\hline Nursing home & - & $14(10.7)$ & $60(68.2)$ & \\
\hline \multicolumn{5}{|l|}{ Smoking habits $\mathrm{N}(\%)$} \\
\hline Nonsmokers & $22(55.0)$ & $102(78.5)$ & $76(90.5)$ & \multirow[t]{2}{*}{$<.001$} \\
\hline Ever smokers & $18(45.0)$ & $28(21.5)$ & $8(9.5)$ & \\
\hline No. cigarettes/d* & $16.1 \pm 8.8(3-40)$ & $15.7 \pm 13.9(2-60)$ & $31.4 \pm 15.7(2-60)$ & $.020^{\dagger}$ \\
\hline Years smoking ${ }^{*}$ & $19.4 \pm 9.1(10-34)$ & $30.4 \pm 18.7(4-66)$ & $29.3 \pm 18.2(6-52)$ & $.154^{\dagger}$ \\
\hline \multicolumn{5}{|l|}{ Nutrition $\mathrm{N}(\%)$} \\
\hline Normal nutrition status & $36(90.0)$ & $106(80.9)$ & 18 (21.7) & \multirow[t]{4}{*}{$<.001$} \\
\hline At risk of malnutrition & $4(10.0)$ & $23(17.6)$ & $53(63.9)$ & \\
\hline Malnourished & - & $2(1.5)$ & $12(14.5)$ & \\
\hline At risk or malnourished & $4(10.0)$ & $25(19.1)$ & $65(70.3)$ & \\
\hline MNA-SF score ${ }^{*}$ & $13.3 \pm 1.4(8-14)$ & $12.8 \pm 1.7(4-14)$ & $9.7 \pm 2.4(4-14)$ & $<.001^{\dagger}$ \\
\hline \multicolumn{5}{|l|}{ Functional status N (\%) } \\
\hline No dependence & $38(95.0)$ & $109(83.2)$ & $5(5.7)$ & \multirow[t]{2}{*}{$<.001$} \\
\hline Dependence & $2(5.0)$ & $22(16.8)$ & $82(94.3)$ & \\
\hline \multicolumn{5}{|l|}{ Comorbidity N (\%) } \\
\hline No comorbidity & $34(85.0)$ & $92(70.2)$ & $52(59.8)$ & \multirow[t]{2}{*}{$.015^{+}$} \\
\hline Comorbidity & $6(15.0)$ & $39(29.8)$ & $35(40.2)$ & \\
\hline
\end{tabular}

ANOVA, analysis of variance; MNA-SF, Mini-Nutritional Assessment-Short Form.

*Mean \pm standard deviation (range).

${ }^{\dagger}$ ANOVA test (bilateral).

${ }^{\ddagger} \chi^{2}$ test (bilateral).

All participants, or their relatives in case of inability, gave informed consent to be included in this study. The study protocol conformed to the principles embodied in the Declaration of Helsinki and was approved by the University of A Coruña Ethics Committee.

\section{Frailty Status}

Frailty status of each participant was determined according to the criteria proposed by Fried et al. ${ }^{11}$ These criteria are based on the presence or absence of 5 specific and phenotypic components (1) unintentional weight loss: at least $4.5 \mathrm{~kg}$ in the past year; (2) self reported exhaustion, identified by 2 questions from the modified 10-item Center for Epidemiological StudiesDepression scale ${ }^{26}$ using the Spanish version ${ }^{27}$; (3)weakness: grip strength in the lowest $20 \%$ at baseline, adjusted for sex and body mass index; (4) slow walking speed: the slowest $20 \%$ at baseline, based on time to walk $4.6 \mathrm{~m}$, adjusting for sex and standing height; and (5) low physical activity: the lowest $20 \%$ at baseline, based on a weighted score of kilocalories expended per week, calculated according to the Spanish validation of the Minnesota Leisure Time Activity questionnaire, ${ }^{28}$ according to each participant's report, and adjusting for sex. Individuals presenting 3 or more of these characteristics were considered frail, while the presence of 1 or 2 of them was considered as a prefrailty state, and the absence of all 5 indicated a nonfrail state. 


\section{Comorbidity}

Charlson comorbidity index ${ }^{25}$ was used to assess general comorbidity and number of comorbid diseases. A Charlson comorbidity index age-adjusted score was computed for each participant, coding the absence of comorbid diseases as 0 , and the presence as 1 to 6 .

\section{Nutritional and Functional Status}

Nutritional screening was carried out with the Mini-Nutritional Assessment-Short Form (MNA-SF) questionnaire ${ }^{29}$ (Spanish version ${ }^{30}$ ), composed of 6 questions from the full MNA questionnaire. ${ }^{31}$ The functional status [ie, the participant's capacity to perform basic activities of daily living (ADL)], was evaluated by an occupational therapist using the Barthel index ${ }^{32}$ validated for Spanish population. ${ }^{33}$

\section{Neopterin Measurement}

Serum neopterin levels were determined by using a commercially available ELISA kit (BRAHMS GmbH, Hennigsdorf, Germany), following the manufacturer's instructions. Sensitivity of the test was $2 \mathrm{nmol} / \mathrm{L}$ neopterin.

\section{Tryptophan, Kynurenine, Phenylalanine, and Tyrosine Analyses}

Serum concentrations of tryptophan and kynurenine on one hand, and plasma concentrations of phenylalanine and tyrosine on the other hand, were simultaneously determined by high performance liquid chromatography with the use of an external albumin-based calibrator and an internal calibrator (3-nitro-L-tyrosine), following the general protocols proposed by Laich et $\mathrm{al}^{34}$ and Neurauter et al, ${ }^{35}$ respectively. The extent of tryptophan breakdown and PHA activity were estimated by calculating the kynurenine to tryptophan ratio (Kyn/Trp) and the phenylalanine to tyrosine ratio (Phe/Tyr), respectively. Limits of detection were $0.1 \mu \mathrm{mol} / \mathrm{L}$ tryptophan, $0.5 \mu \mathrm{mol} / \mathrm{L}$ kynurenine, and $0.3 \mu \mathrm{mol} / \mathrm{L}$ phenylalanine and tyrosine.

\section{Nitrite Determination}

The stable $\mathrm{NO}$ metabolite nitrite $\left(\mathrm{NO}_{2^{-}}\right)$was measured in plasma samples as an estimation of NOS activity and NO production, ${ }^{36}$ according to the Griess method. A standard curve was prepared with different $\mathrm{NaNO}_{2}$ concentrations; then $50 \mathrm{~mL}$ of plasma or standard curve samples and $125 \mu \mathrm{L}$ of Griess reagent (Merck, Darmstadt, Germany) were added onto a microplate. After 10 minutes of incubation at room temperature without shaking, color development was measured at $562 \mathrm{~nm}$ in a power wave X microplate reader (Bio-Tek Instruments, Winooski, VT), equipped with kinetic analysis software (KC4 v.2.5; Bio-Tek Instruments). Limit of detection was 1.5 $\mu \mathrm{mol} / \mathrm{L}$ nitrite.

\section{Statistical Analysis}

A general description of the study population was conducted by univariate analysis, comparing sociodemographic features (ie, sex, age, and living conditions), lifestyle factors (ie, tobacco consumption, nutritional status), and several clinical factors (ie, functional status, comorbidity) in the 3 groups of older adults classified according to their frailty status (nonfrail, prefrail, and frail). Analysis of variance was applied for continuous variables and c2 test was used for categorical variables.

Preliminary univariate analyses through analysis of variance and Tuckey post-hoc test were performed to assess the effect of frailty status on the immune biomarkers. The effect of nutritional and functional status was preliminarily evaluated by Student $t$ test. Data from tryptophan, 
kynurenine, and tyrosine followed a normal distribution (Kolmogorov-Smirnov goodness-of-fit test). To achieve a better approximation to the normal distribution, a log-transformation of the data was applied to Kyn/Trp ratio. As no improvement was achieved with transformation, the KruskalWallis and Mann-Whitney U tests were applied for statistical evaluation of neopterin, nitrite, phenylalanine concentrations, and Phe/Tyr ratios.

Reference ranges were calculated for the immune biomarkers on the basis of values from nonfrail and prefrail individuals. For those biomarkers following a normal distribution, reference ranges were defined by the mean \pm 2 standard deviation. When data were considered to have a non-Gaussian distribution, reference ranges were defined as the central $95 \%$ of the area under the distribution curve (from $2.5 \%$ to $97.5 \%$ ).

Linear regression models were applied on the log-transformed data to estimate the effect of frailty status, nutritional status, and functional status. All models included sex, age, and smoking habits (never/ever smokers). The results are presented as mean ratios and $95 \%$ confidence intervals.

Associations between immune biomarkers were estimated by partial correlation coefficients, adjusting for sex, age, and smoking habits. The critical limit for significance was set at $P<.05$. Analyses were carried out using the IBM SPSS software package V. 20 (SPSS, Inc, Chicago, IL) and the STATA/SE software package V. 12.0 (StataCorp LP, College Station, TX).

\section{Results}

The population analyzed (Table 1) was composed of 259 participants, mainly female participants (67\%), age range 65-102 years. The prefrail group was the most numerous, followed by frail participants. Among all 131 prefrail participants, $89(68 \%)$ were positive for only 1 frailty criterion, and $42(32 \%)$ were positive for 2 frailty criteria. The low grip strength criterion, indicative of muscle weakness, was present in most prefrail individuals $(\mathrm{N}=126,96 \%)$. Most frail patients lived in nursing homes, whereas all nonfrail participants lived at family home, not attending daycare centers. Prevalence of smoking habits was quite balanced in the group of nonfrail participants, but nonsmokers were clearly more frequent in the other 2 groups. Regarding nutrition, few individuals were malnourished, most of them frail and none nonfrail; accordingly the mean MNA-SF score was lower in frail participants. Only 2 nonfrail participants were classified as ADL dependent, according to their functional status, whereas most frail patients were ADL dependent. Moreover, comorbidity was present in only $15 \%$ of nonfrail participants, but in $40 \%$ of frail individuals.

Results of the immune biomarkers analyzed in the nonfrail, prefrail, and frail groups are shown in Table 2. According to univariate analyses, significant and progressive changes were observed in concentrations of several biomarkers. Significant increases were obtained of neopterin concentrations and Kyn/Trp ratio in the frail group with regard to the other 2 groups. On the contrary, tryptophan, nitrite and tyrosine levels decreased significantly in the presence of frailty; only in the case of nitrite were the 3 population groups significantly different. 
Table 2. Results of Immune Biomarkers in the Study Group, Classified According to Frailty Status (Univariate Analysis)

\begin{tabular}{|c|c|c|c|c|}
\hline \multirow[t]{2}{*}{ Immune Biomarker } & Nonfrail & Prefrail & Frail & \multirow[t]{2}{*}{$P$ Value* } \\
\hline & $\mathrm{N}($ Mean $\pm \mathrm{SE})$ & $\mathrm{N}($ Mean $\pm \mathrm{SE})$ & $\mathrm{N}($ Mean $\pm \mathrm{SE})$ & \\
\hline Neopterin (nmol/L) & $37\left(5.96 \pm 0.30^{\mathrm{a}}\right)$ & $120\left(7.53 \pm 0.44^{\mathrm{a}}\right)$ & $86\left(13.04 \pm 0.85^{\mathrm{b}}\right)$ & $<.001$ \\
\hline Tryptophan $(\mu \mathrm{mol} / \mathrm{L})$ & $35\left(82.30 \pm 2.52^{a}\right)$ & $118\left(75.72 \pm 1.42^{\mathrm{a}}\right)$ & $86\left(62.71 \pm 1.64^{b}\right)$ & $<.001$ \\
\hline Kynurenine $(\mu \mathrm{mol} / \mathrm{L})$ & $35(2.26 \pm 0.09)$ & $118(2.44 \pm 0.07)$ & $86(2.41 \pm 0.08)$ & .467 \\
\hline $\mathrm{Kyn} / \operatorname{Trp}(\mu \mathrm{mol} / \mathrm{mmol})$ & $35\left(37.60 \pm 3.20^{\mathrm{a}}\right)$ & $118\left(42.93 \pm 2.14^{\mathrm{a}}\right)$ & $86\left(81.70 \pm 3.98^{\mathrm{b}}\right)$ & $<.001$ \\
\hline Nitrite $(\mu \mathrm{mol} / \mathrm{L})$ & $37\left(13.09 \pm 2.66^{\mathrm{a}}\right)$ & $121\left(8.16 \pm 0.79^{b}\right)$ & $77\left(1.90 \pm 0.41^{\mathrm{c}}\right)$ & $<.001$ \\
\hline Tyrosine $(\mu \mathrm{mol} / \mathrm{L})$ & $37\left(112.21 \pm 4.98^{\mathrm{a}}\right)$ & $123\left(108.85 \pm 2.80^{\mathrm{a}}\right)$ & $86\left(96.34 \pm 2.37^{b}\right)$ & .002 \\
\hline Phenylalanine $(\mu \mathrm{mol} / \mathrm{L})$ & $37(79.56 \pm 3.54)$ & $123(73.26 \pm 1.51)$ & $86(76.52 \pm 3.56)$ & .336 \\
\hline Phe/Tyr $(\mu \mathrm{mol} / \mu \mathrm{mol})$ & $37(0.72 \pm 0.02)$ & $123(0.70 \pm 0.01)$ & $86(0.81 \pm 0.04)$ & .061 \\
\hline
\end{tabular}

Note. Bold values are statistically significant $(P<.05)$

ANOVA, Analysis of variance.

Different superscript letters (a, b, c) indicate statistically different groups (Tukey test or Mann-Whitney U test)

*Multiple group comparison (ANOVA or Kruskal-Wallis test).

Because reference ranges specific for robust older adults were not available for any of the biomarkers analyzed in this work, values obtained from nonfrail and prefrail participants were used for calculating the lower and upper limits of the corresponding reference ranges (Table 3), since no significant differences between these 2 groupswere observed, with the exception of nitrite. Percentages of concentrations registered in frail participants out of the calculated reference ranges were notable for Kyn/Trp ratio (above) and tryptophan (below), and moderate for neopterin (above) and nitrite (below). Values exceeding the reference range in both directions were observed for phenylalanine and Phe/Tyr ratio.

Table 3. Reference Ranges of the Immune Biomarkers Analyzed, Calculated on the Basis of Results Obtained in Nonfrail and Prefrail Participants

\begin{tabular}{|c|c|c|c|c|c|c|}
\hline \multirow[t]{2}{*}{ Immune Biomarker } & \multirow[t]{2}{*}{$N$} & \multirow[t]{2}{*}{$\begin{array}{l}\text { Values in } \\
\text { "Healthy" } \\
\text { Participants* }\end{array}$} & \multicolumn{2}{|c|}{ Reference Range } & \multicolumn{2}{|c|}{$\begin{array}{l}\text { Percent of Frail } \\
\text { Participants Out of the } \\
\text { Reference Range }\end{array}$} \\
\hline & & & $\begin{array}{l}\text { Lower } \\
\text { Limit }\end{array}$ & $\begin{array}{l}\text { Upper } \\
\text { Limit }\end{array}$ & Below & Above \\
\hline Neopterin (nmol/L) & 157 & $5.94(1.58)$ & 3.50 & 22.40 & - & 10.5 \\
\hline Tryptophan $(\mu \mathrm{mol} / \mathrm{L})$ & 153 & $67.4(10.2)$ & 46.14 & 108.30 & 16.3 & - \\
\hline Kynurenine $(\mu \mathrm{mol} / \mathrm{L})$ & 153 & $1.78(0.42)$ & 0.88 & 3.92 & - & 3.5 \\
\hline $\mathrm{Kyn} / \operatorname{Trp}(\mu \mathrm{mol} / \mathrm{mmol})$ & 153 & $26.7(6.2)$ & 16.69 & 99.43 & - & 22.1 \\
\hline Nitrite $(\mu \mathrm{mol} / \mathrm{L})$ & 158 & $44.9(32.0)$ & 0.59 & 36.19 & 9.1 & - \\
\hline Tyrosine $(\mu \mathrm{mol} / \mathrm{L})$ & 160 & $90.6(22.9)$ & 47.95 & 171.31 & - & 1.2 \\
\hline Phenylalanine $(\mu \mathrm{mol} / \mathrm{L})$ & 160 & $65.2(11.1)$ & 48.11 & 118.72 & 5.8 & 7.0 \\
\hline Phe/Tyr & 160 & $0.75(0.14)$ & 0.36 & 1.05 & 1.2 & 9.3 \\
\hline
\end{tabular}

*According to Geisler et $\mathrm{al}^{37}$ (2015) (mean age \pm standard deviation: $49 \pm 11.4$ years).

Remarkable significant correlations were obtained between biomarkers (Table 4). Neopterin showed strong associations with tryptophan breakdown parameters and slight association with nitrite and Phe/Tyr. In turn, nitrite and phenylalanine metabolism products were moderately associated with tryptophan breakdown products. Frailty presented significant direct associations with neopterin, Kyn/Trp and Phe/Tyr, and inverse associations with tryptophan, nitrite, and tyrosine. 
Table 4. Partial Correlation Coefficients Between Biomarkers Analyzed, Adjusted by Age, Sex and Smoking Habits (Cells in Light Gray: Moderate Associations, Cell in Dark Gray: Strong Association)

\begin{tabular}{|c|c|c|c|c|c|c|c|c|}
\hline Biomarker & Neopterin & Tryptophan & Kynurenine & Kyn/Trp & Nitrite & $\begin{array}{l}\text { Phenyl- } \\
\text { alanine }\end{array}$ & Tyrosine & Phe/Tyr \\
\hline Frailty & $0.209 * *$ & $-0.294 * * *$ & -0.070 & $0.344 * * *$ & $-0.328 * * *$ & -0.049 & $-0.223 * *$ & $0.162 *$ \\
\hline Neopterin & & -0.233 & $0.365 * * *$ & $0.565^{* * *}$ & $-0.219 * *$ & 0.038 & -0.122 & $0.151^{*}$ \\
\hline Tryptophan & & & $0.268 * * *$ & & 0.119 & $0.304 * * *$ & $0.410 * * *$ & -0.094 \\
\hline Kynurenine & & & & & -0.031 & 0.080 & $0.167 *$ & -0.078 \\
\hline Kyn/Trp & & & & & $-0.367 * * *$ & -0.041 & $-0.134 *$ & 0.086 \\
\hline Nitrite & & & & & & -0.055 & -0.074 & 0.039 \\
\hline Phenylalanine & & & & & & & $0.491 * * *$ & \\
\hline
\end{tabular}

$* P<.05 ; * * P<.01, * * * P<.001$.

Table 5 summarizes the results from the multivariate statistical analyses. All models were significant and adjusted by age, sex, and smoking habits. Results were essentially in agreement with those obtained from the univariate analyses (ie, significant increases in neopterin and Kyn/Trp, together with Phe/Tyr levels, and significant decreases in tryptophan, nitrite, and tyrosine concentrations in frail individuals compared with nonfrail participants). No significant differences were observed between nonfrail and prefrail participants, except in the case of nitrite concentrations, which showed a progressive decline with increasing frailty severity. Significant positive influence of age was obtained in neopterin, kynurenine, and Kyn/Trp levels, and inverse influence was observed in tryptophan concentrations.

Table 5. Effect of Frailty Status on Immunologic Biomarkers; Models Adjusted by Age, Sex, and Smoking Habits

\begin{tabular}{|c|c|c|c|c|}
\hline \multirow[t]{2}{*}{$\begin{array}{l}\text { Frailty Status } \\
\text { Group }\end{array}$} & Neopterin & Tryptophan & Kynurenine & Kyn/Trp \\
\hline & $\begin{array}{l}\text { Mean Ratio (95\% } \\
\text { CI) }\end{array}$ & $\begin{array}{l}\text { Mean Ratio (95\% } \\
\text { CI) }\end{array}$ & $\begin{array}{l}\text { Mean Ratio (95\% } \\
\text { CI) }\end{array}$ & $\begin{array}{l}\text { Mean Ratio }(95 \% \\
\text { CI) }\end{array}$ \\
\hline \multicolumn{5}{|l|}{ Frailty status } \\
\hline Nonfrail & 1.00 & 1.00 & 1.00 & 1.00 \\
\hline Prefrail & $1.06(0.88-1.27)$ & $0.93(0.85-1.01)$ & $1.03(0.91-1.16)$ & $1.08(0.91-1.29)$ \\
\hline \multirow[t]{3}{*}{ Frail } & $1.41(1.15-1.76)^{* *}$ & $0.81(0.73-0.89) * *$ & $0.94(0.81-1.08)$ & $1.76(1.43-2.17)^{* * *}$ \\
\hline & Nitrite & Tyrosine & Phenylalanine & Phe/Tyr \\
\hline & $\begin{array}{l}\text { Mean Ratio (95\% } \\
\text { CI) }\end{array}$ & $\begin{array}{l}\text { Mean Ratio (95\% } \\
\text { CI) }\end{array}$ & $\begin{array}{l}\text { Mean Ratio (95\% } \\
\text { CI) }\end{array}$ & $\begin{array}{l}\text { Mean Ratio (95\% } \\
\text { CI) }\end{array}$ \\
\hline \multicolumn{5}{|l|}{ Frailty status } \\
\hline Nonfrail & 1.00 & 1.00 & 1.00 & 1.00 \\
\hline Prefrail & $0.65(0.44-10.97) *$ & $0.94(0.85-1.04)$ & $0.93(0.84-1.04)$ & $1.00(0.90 \mathrm{e} 1.10)$ \\
\hline Frail & $0.18(0.11-0.30) * *$ & $0.82(0.73-0.92) * *$ & $0.92(0.81-1.04)$ & $1.12(1.00-1.26)^{*}$ \\
\hline
\end{tabular}

Note. Bold values are statistically significant $(P<.05)$

CI, confidence interval.

$* P<.05 ; * * P<.01$

Nutritional status and functional status were also shown to significantly influence some biomarkers (Table 6). Specifically, individuals malnourished or at risk of malnutrition presented significantly higher levels of neopterin, Kyn/Trp, and Phe/Tyr, and significantly lower concentrations of tryptophan, nitrite, and tyrosine (borderline significant $P=.053$, in the last case) than participants with normal nutrition. Similar results were obtained in ADL dependent patients compared with nondependent individuals. 
Table 6. Effect of Nutritional Status and Functional Status on Immunologic Biomarkers; Models Adjusted by Age, Sex, and Smoking Habits

\begin{tabular}{|c|c|c|c|c|}
\hline \multirow[t]{2}{*}{ Group } & Neopterin & Tryptophan & Kynurenine & Kyn/Trp \\
\hline & $\begin{array}{l}\text { Mean Ratio }(95 \% \\
\text { CI) }\end{array}$ & $\begin{array}{l}\text { Mean Ratio (95\% } \\
\text { CI) }\end{array}$ & $\begin{array}{l}\text { Mean Ratio (95\% } \\
\text { CI) }\end{array}$ & $\begin{array}{l}\text { Mean Ratio }(95 \% \\
\text { CI) }\end{array}$ \\
\hline \multicolumn{5}{|l|}{ Nutritional status } \\
\hline Normal nutrition & 1.00 & 1.00 & 1.00 & 1.00 \\
\hline $\begin{array}{l}\text { At risk or } \\
\text { malnourished } \\
\text { Functional status }\end{array}$ & $1.22(1.07-1.39)^{* * *}$ & $0.92(0.87-0.98)^{* * *}$ & $0.96(0.88-1.04)$ & $1.30(1.36-1.48)^{* * *}$ \\
\hline Nondependent & 1.00 & 1.00 & 1.00 & 1.00 \\
\hline \multirow[t]{3}{*}{ Dependent } & $1.40(1.21-1.61)^{* * *}$ & $0.89(0.83-0.95)^{* * *}$ & $0.99(0.90-1.10)$ & $1.77(1.55-2.02)^{* *}$ \\
\hline & Nitrite & Tyrosine & Phenylalanine & Phe/Tyr \\
\hline & $\begin{array}{l}\text { Mean Ratio (95\% } \\
\text { CI) }\end{array}$ & $\begin{array}{l}\text { Mean Ratio (95\% } \\
\text { CI) }\end{array}$ & $\begin{array}{l}\text { Mean Ratio (95\% } \\
\text { CI) }\end{array}$ & $\begin{array}{l}\text { Mean Ratio (95\% } \\
\text { CI) }\end{array}$ \\
\hline \multicolumn{5}{|l|}{ Nutritional status } \\
\hline Normal nutrition & 1.00 & 1.00 & 1.00 & 1.00 \\
\hline $\begin{array}{l}\text { At risk or } \\
\text { malnourished } \\
\text { Functional status }\end{array}$ & $0.47(0.34-0.65)^{* * *}$ & $0.93(0.87-1.00) \dagger$ & $1.03(0.96-1.11)$ & $1.11(1.03 \mathrm{e} 1.19) * *$ \\
\hline Nondependent & 1.00 & 1.00 & 1.00 & 1.00 \\
\hline Dependent & $0.27(0.19-0.38)^{* *}$ & $0.88(0.81-0.96)^{* *}$ & $0.97(0.89-1.06)$ & $1.10(1.02-1.19) *$ \\
\hline
\end{tabular}

Note. Bold values are statistically significant $(P<.05)$

CI, confidence interval.

$* P<.05 ; * * P<.01$.

$\dagger P=.053$.

\section{Discussion}

A huge body of evidence supports the age-related chronic lowgrade inflammation (inflammaging). ${ }^{38-40}$ Further, chronic low-grade inflammation is considered to be involved in agerelated maladies, such as neurodegenerative diseases, ${ }^{41}$ cardiovascular diseases, ${ }^{42}$ osteoporosis, ${ }^{43}$ or cancer. ${ }^{44}$ Associations between several proinflammatory factors, namely interleukin 6, C reactive protein, and tumor necrosis factor alpha, and frailty have been previously reported. ${ }^{45-48}$ Besides, purified monocytes from frail participants were reported to have upregulated expression of stress-responsive inflammatory pathway genes, compared with monocytes from nonfrail individuals, in ex vivo studies with or without lipopolysaccharide stimulation. ${ }^{49,50}$ Still, data on in vivo immune stimulation and monocyte/macrophage activity related to frailty status are very scarce. To our knowledge, no studies addressed so far the possible relationship of frailty status with immunologic biomarkers involved in GCH or IDO enzymatic pathways, except for neopterin. $^{51,52}$ Hence, the possible disturbance of the mentioned immune stimulation-related enzymatic pathways was analyzed in the present work in a population of Spanish older adults, classified according to their frailty status following the 5 phenotypic criteria proposed by Fried et al. $^{11}$

Although concentrations of the immune biomarkers assessed in this work were previously reported in populations or subpopulations of older adults, ${ }^{53-58}$ frailty status of the participants in these studies was not determined; at most some reports specified they were "healthy." Thus, it was necessary to establish reference ranges of these biomarkers specifically in the group of robust older adults (ie, in the absence of frailty). For some of the immune biomarkers, namely neopterin, nitrite, and especially Kyn/Trp and tryptophan, the rate of concentrations in the frail group out of the reference range was remarkable and entirely in the same direction (only above reference range in the case of neopterin and Kyn/Trp, and only below range in tryptophan and nitrite), indicating a clear tendency of disturbance related to frailty status. 
Neopterin concentration in body fluids is considered as a marker of activation of the immune system, in particular of Th1 or cell-mediated response. ${ }^{2} 3$ Higher concentrations of neopterin in older age were previously reported, ${ }^{51,58,59}$ and association between increased neopterin concentrations and enhanced tryptophan breakdown (as indicated by Kyn/Trp ratio) has been documented in older adults as well. ${ }^{54,60-62}$ Similarly, our results show significant and positive influence of age on neopterin and kynurenine concentrations and $\mathrm{Kyn} / \mathrm{Trp}$ ratio, and inverse influence on tryptophan levels.

To date, only very few studies evaluated neopterin serum levels in older adults in association with frailty, finding significantly higher neopterin concentrations in frail older adults than in nonfrail controls, either equally older ${ }^{51}$ or younger (median 38 years). ${ }^{52}$ Current results support those previous ones and also indicate association of frailty with tryptophan breakdown because significant influence was observed for frailty status on neopterin, tryptophan, and Kyn/Trp ratio. Moreover, our results are also in line with other studies reporting that neopterin urinary concentrations and $\mathrm{Kyn} / \mathrm{Trp}$ ratio predict mortality in nonagenarians ${ }^{60,61}$ because frailty is related to increased vulnerability to stressors and increases the risk of death ${ }^{4,63}$

Alterations of Kyn/Trp ratio may be due to an enhanced activity of 2 enzymes, namely IDO and tryptophan 2,3-dioyxgenase (TDO), an IDO isoenzyme not induced by proinflammatory cytokines but rather upregulated by tryptophan itself and corticosteroids. ${ }^{64,65}$ However, in the presence of immune stimulation, Kyn/Trp together with concentrations of neopterin reflect the degree of Th1-type immune activation. ${ }^{66}$ The strong correlation found in this study between neopterin concentration and Kyn/Trp points to enhanced IDO activity and immune stimulation as the cause behind tryptophan parameters disturbance. Besides, in many cases, the tryptophan breakdown rate not only correlates with neopterin concentrations, but also with the extent and the activity of the disease (eg, in viral infections or malignant tumors). ${ }^{20,67,68}$ The moderate significant correlations obtained in the current study between frailty status and tryptophan breakdown parameters, and also with nitrite and tyrosine, suggest that the level of these markers may be indicative (directly or inversely) of frailty severity. This also indicates that, although neopterin and tryptophan breakdown products are not specific biomarkers for frailty, development of frailty status takes most likely place when these immune biomarkers increase, being the immune system activation a strong driving force for frailty development.

Activated human monocytes/macrophages produce neopterin at the expense of $\mathrm{BH} 4 .{ }^{69} \mathrm{BH} 4$ deficiency affects PHA and NOS enzymatic activities, consequently diminishing tyrosine and NO production and increasing the ratio Phe/Tyr, considered a useful measure to estimate $\mathrm{PAH}$ activity. ${ }^{70}$ Indeed, increases in phenylalanine concentration and in Phe/Tyr have been reported in patients with different chronic inflammatory conditions, and correlations with neopterin concentrations were also found..$^{21,71-73}$ Our results showed a significant influence of frailty status on Phe/Tyr (direct) and on tyrosine and nitrite concentrations (inverse), supporting the view that both PAH and NOS activities are impaired in frail older adults. The significant correlation found between Phe/Tyr and neopterin, and the inverse associations observed of nitrite with neopterin concentrations and Kyn/Trp, also point toward parallel disturbance of GCH and IDO enzymatic pathways caused by Th1-type immune activation in frail older adults.

No significant association was obtained in this work between nitrite and any of the phenylalanine breakdown parameters. Several reasons may help to explain this lack of association. On one hand, even though the majority of plasma nitrite is derived from constitutive NOS activity, ${ }^{36}$ serum nitrite concentrations only serve as a rough estimate of NO production rates ${ }^{71}$; indeed food is an important exogenous factor influencing serum nitrite concentrations. ${ }^{37}$ And, on the other hand, tyrosine is not an end-product and its concentrations are also influenced by the activity of another $\mathrm{BH}_{4}$-dependent enzyme (tyrosine hydroxylase), which forms L-DOPA (L-3,4dihydroxyphenylalanine) from tyrosine. ${ }^{22}$

In elderly persons, nutrition-related problems are very common. In the study population, $14.5 \%$ of frail peoplewere malnourished and $90 \%$ of participants at risk or malnourished were prefrail or frail. In addition, 93\% frail participants were dependent to perform ADL activities. When the potential influence of nutritional status or functional status on the immune biomarkers was tested in the current study, results obtained were parallel to those for frailty status. This was not surprising because an association and overlap between frailty and impaired nutritional status has 
been previously demonstrated, revealing that patients who are at risk of malnutrition are more likely to be frail and have impaired mobility. ${ }^{74}$ Besides, a 12 -year prospective population-based study reported that some particularly unhealthy dietary patterns may increase the risk of frailty in older adults. ${ }^{75}$ And a recent meta-analysis revealed that malnutrition and physical frailty in community-dwelling older adults are related, but prevalence of physical frailty is much higher than the prevalence of malnutrition (19\% vs $2.3 \%$, respectively), indicating that these syndromes are not interchangeable. ${ }^{76}$

Moreover, significant relationship between frailty and functional status (disabilities in instrumental ADL scale, which precede disabilities in ADL and loss of autonomy)was reported. ${ }^{77}$ And it has also been shown that malnutrition compromises the functional status. ${ }^{78}$ Hence, our results show the interrelationship between frailty, nutritional status, and functional status; still, frailty is a more a holistic concept involving not only nutritional and functional features, but also a general physiological decline.

Besides, age-related decline of the immune system can undermine the dynamic homeostatic balance between the microbiota and the gut-associated immune system, leading to changes in intestinal microbial structure and composition. ${ }^{79}$ Indeed, immunosenescence and inflammaging have been connected to changes in microbiota composition in older adults. ${ }^{80,81}$ Moreover, alterations in the gut microbiota composition have been associated with several chronic conditions, including frailty. ${ }^{82-84}$ The composition of the microbiome will certainly influence the degree of immune system-derived metabolic alterations. Immune response strongly affects biochemical pathways that are most relevant in the control of pathogens growth. Thus, restricting availability of the essential amino acid tryptophan is of great relevance limiting protein biosynthesis, but it also interferes with the biochemistry of tryptophan for serotonin production and the kynurenine pathway (thus, affecting mood). Therefore, microbiome composition can play a role in the relationship between frailty and the immune stimulation biomarkers analyzed in this study, but further investigation is required to explore this possibility.

In summary, results obtained in the present study are consistent with the idea that chronic immune system stimulation in frail older adults is higher than expected according only to their age (ie, frailty status in the elderly is associated with an additional degree of immune stimulation, manifested in more intense disturbance of IDO and $\mathrm{GCH}$ pathways than in nonfrail or prefrail older adults). Nevertheless, because this study was carried out in an older adult population, a major limitation is that participants are not completely healthy, but most of them present different pathologic conditions (in some cases comorbidity), and take medications to treat them. Although exclusion criteria adopted included having autoimmune diseases, neoplasia or any chronic infection, and taking medications known to affect the immune system, it is possible that some of the chronic diseases that are usually found in older adults modify the levels of immune systemrelated molecules.

\section{Conclusions}

This work establishes, for the first time, reference ranges for a number of immune biomarkers related to IDO and GCH enzymatic pathways in the population of robust older adults (ie, excluding the presence of frailty).

Furthermore, results from this study provide evidence for the existence of significant influence of frailty status on circulating concentrations of immune biomarkers involved in IDO and GCH enzymatic pathways. Significant correlations observed between immune biomarkers indicate they change in parallel, not independently, thus, pointing to interrelated causes. Altogether these results suggest that the presence and intensity of immune stimulation in frail participants is not only due to their age itself. In other words, our data support the involvement of monocyte/macrophage mediated Th1 immune activation and disturbed amino acid biochemistry in the pathophysiology of the frailty geriatric syndrome.

These findings provide a basis for further investigations on the underlying immune mechanisms that contribute to frailty status in the elderly to determine the orientation and feasibility of future interventional strategies focused on prevention and treatment of frailty. 


\section{References}

1. United Nations, Department of Economic and Social Affairs, Population Division. World Population Prospects: The 2015 Revision. Available at:

http://esa.un.org/unpd/wpp/index.htm. Accessed May 3, 2017.

2. Romero-Ortuno, R. Frailty in primary care. Interdiscip Top Gerontol Geriatr. 2015;41:8594.

3. Cesari, M., Gambassi, G., van Kan, G.A. et al, The frailty phenotype and the frailty index: Different instruments for different purposes. Age Ageing. 2014;43:10-12.

4. Morley, J.E., Vellas, B., Abellan van Kan, G. et al, Frailty consensus: A call to action. J Am Med Dir Assoc. 2013;14:392-397.

5. Ahmed, N., Mandel, R., Fain, M.J. Frailty: An emerging geriatric syndrome. Am J Med. 2007;120:748-753.

6. Rockwood, K., Mitnitski, A. Frailty in relation to the accumulation of deficits. J Gerontol A Biol Sci Med Sci. 2007;62:722-727.

7. Walston, J., Hadley, E.C., Ferrucci, L. et al, Research agenda for frailty in older adults: Toward a better understanding of physiology and etiology: Summary from the American Geriatrics Society/National Institute on Aging research conference on frailty in older adults. J Am Geriatr Soc. 2006;54:991-1001.

8. Collard, R.M., Boter, H., Schoevers, R.A. et al, Prevalence of frailty in communitydwelling older persons: A systematic review. J Am Geriatr Soc. 2012;60:1487-1492.

9. García-García, F.J., Gutiérrez Ávila, G., Alfaro Acha, A. et al, The prevalence of frailty syndrome in an older population from Spain. The Toledo Study for Healthy Aging. J Nutr Health Aging. 2011;15:852-856.

10. Abizanda, P., Sánchez-Jurado, P.M., Romero, L. et al, Prevalence of frailty in a Spanish elderly population: The Frailty and Dependence in Albacete study. J Am Geriatr Soc. 2011;59:1356-1359.

11. Fried, L.P., Tangen, C.M., Walston, J. et al, Cardiovascular Health Study Collaborative Research Group. Frailty in older adults: Evidence for a phenotype. J Gerontol A Biol Sci Med Sci. 2001;56:M146-M156.

12. González-Vaca, J., De La Rica-Escuín, M., Silva-Iglesias, M. Frailty in institutionalized older adults from Albacete. The FINAL Study: Rationale, design, methodology, prevalence and attributes. Maturitas. 2014;77:78-84.

13. Libby, P. Inflammation in atherosclerosis. Nature. 2002;420:868-874.

14. Pradhan, A.D., Manson, J.E., Rifai, N. et al, C-reactive protein, interleukin 6, and risk of developing type 2 diabetes mellitus. JAMA. 2001;286:327-334.

15. Griffin, W.S. Inflammation and neurodegenerative diseases. Am J Clin Nutr. 2006;83:470-474.

16. Faienza, M.F., Ventura, A., Marzano, F., Cavallo, L. Postmenopausal osteoporosis: The role of immune system cells. Clin Dev Immunol. 2013;2013:575936.

17. Castelo-Branco, C., Soveral, I. The immune system and aging: A review. Gynecol Endocrinol. 2014;30:16-22.

18. Franceschi, C., Capri, M., Monti, D. Inflammaging and anti-inflammaging: A systemic perspective on aging and longevity emerged from studies in humans. Mech Ageing Dev. 2007; 128:92-105.

19. Widner, B., Werner, E.R., Schennach, H. et al, Simultaneous measurement of serum tryptophan and kynurenine by HPLC. Clin Chem. 1997;43:2424-2426.

20. Schröcksnadel, K., Wirleitner, B., Winkler, C., Fuchs, D. Monitoring tryptophan metabolism in chronic immune activation. Clin Chim Acta. 2006;364:82-90.

21. Neurauter, G., Schrocksnadel, K., Scholl-Burgi, S. et al, Chronic immune stimulation correlates with reduced phenylalanine turnover. Curr Drug Metab. 2008;9:622-627.

22. Widner, B., Ledochowski, M., Fuchs, D. Interferon-gamma-induced tryptophan degradation: Neuropsychiatric and immunological consequences. Curr Drug Metab. 2000;1:193-204.

23. Murr, C., Widner, B., Wirleitner, B., Fuchs, D. Neopterin as a marker for immune system activation. Curr Drug Metab. 2002;3:175-187.

24. Geisler, S., Gostner, J.M., Becker, K. et al, Immune activation and inflammation increase the plasma phenylalanine-to-tyrosine ratio. Pteridines. 2013;24:27-31.

25. Charlson, M.E., Pompei, P., Ales, K.L., MacKenzie, C.R. A new method of classifying prognostic comorbidity in longitudinal studies: Development and validation. J Chronic Dis. 1987;40:373-383.

26. Radloff, L.S. The CES-D scale: A self-report depression scale for research in the general population. App Psychol Meas. 1977;1:385-401.

27. Ruiz-Grosso, P., Loret de Mola, C., Vega-Dienstmaier, J.M. et al, Validation of the Spanish Center for Epidemiological Studies Depression and Zung Self-Rating Depression Scales: A comparative validation study. PloS One. 2012;7:e45413. 
28. Ruiz-Comellas, A., Pera, G., Baena Díez, J.M. et al, Validación de una versión reducida en español del cuestionario de actividad física en el tiempo libre de Minnesota. Rev Esp Salud Pública. 2012;86:495-508.

29. Kaiser, M.J., Bauer, J.M., Ramsch, C. et al, MNA-International Group. Validation of the Mini nutritional assessment short-form (MNA-SF): A practical tool for identification of nutritional status. J Nutr Health Aging. 2009;13:782-788.

30. Nestlé Nutrition Institute. Guía para rellenar el formulario Mini Nutritional Assessment (MNA®). ; 2009 (Available at:) http://www.mna-elderly.com/mna_forms.html (Accessed May 3, 2017).

31. Guigoz, Y., Vellas, B.J., Garry, P.J. Mini-nutritional Assessment (MNA): A practical assessment tool for grading the nutritional state of elderly patients. in: B.J. Vellas, Y. Guigoz, P.J. Garry, J.L. Albarede (Eds.) Facts and Research in Gerontology. Serdi Publishing Co, New York; 1994:15-60.

32. Mahoney, F.I., Barthel, D.W. Functional evaluation: The Barthel index. Maryland State Med J. 1965;14:61-65.

33. Baztán, J.J., Pérez del Molino, J., Alarcón, T. et al, Índice de Barthel: Instrumento válido para la valoración funcional de pacientes con enfermedad cerebrovascular. Rev Esp Geriatr Gerontol. 1993;28:32-40.

34. Laich, A., Neurauter, G., Widner, B., Fuchs, D. More rapid method for simultaneous measurement of tryptophan and kynurenine by HPLC. Clin Chem. 2002;48:579-581.

35. Neurauter, G., Scholl-Bürgi, S., Haara, A. et al, Simultaneous measurement of phenylalanine and tyrosine by high performance liquid chromatography (HPLC) with fluorescence detection. Clin Biochem. 2013;46:1848-1851.

36. Kleinbongard, P., Dejam, A., Lauer, T. et al, Plasma nitrite reflects constitutive nitric oxide synthase activity in mammals. Free Radic Biol Med. 2003;35:790-796.

37. Geisler, S., Mayersbach, P., Becker, K. et al, Serum tryptophan, kynurenine, phenylalanine, tyrosine and neopterin concentrations in 100 healthy blood donors. Pteridines. 2015;26:31-36.

38. Franceschi, C., Bonafè, M., Valensin, S. et al, Inflamm-aging. An evolutionary perspective on immunosenescence. Ann NY Acad Sci. 2000;908:244-254.

39. Franceschi, C., Campisi, J. Chronic inflammation (inflammaging) and its potential contribution to age-associated diseases. J Gerontol A Biol Sci Med Sci. 2014;69:S4-S9.

40. Sergio, G. Exploring the complex relations between inflammation and aging (inflammaging): Anti-inflamm-aging remodelling of inflamm- aging, from robustness to frailty. Inflamm Res. 2008;57:558-563.

41. Glass, C.K., Saijo, K., Winner, B. et al, Mechanisms underlying inflammation in neurodegeneration. Cell. 2010;140:918-934.

42. Libby, P., Okamoto, Y., Rocha, V.Z., Folco, E. Inflammation in atherosclerosis: Transition from theory to practice. Circ J. 2010;74:213-220.

43. Lencel, P., Magne, D. Inflammaging: The driving force in osteoporosis?. Med Hypotheses. 2011;76:317-321.

44. Thun, M.J., Henley, S.J., Gansler, T. Inflammation and cancer: An epidemiological perspective. Novartis Found Symp. 2004;256:6-21 (discussion 22-28, 49-52, 266-269).

45. Barzilay, J.I., Blaum, C., Moore, T. et al, Insulin resistance and inflammation as precursors of frailty: The Cardiovascular Health Study. Arch Intern Med. 2007;167:635641.

46. Hubbard, R.E., O’Mahony, M.S., Savva, G.M. et al, Inflammation and frailty measures in older people. J Cell Mol Med. 2009;13:3103-3109.

47. Leng, S.X., Xue, Q.L., Tian, J. et al, Associations of neutrophil and monocyte counts with frailty in community-dwelling disabled older women: Results from the Women's Health and Aging Studies I. Exp Gerontol. 2009;44:511-516.

48. Rooning, B., Wyller, T.B., Seljeflot, I. et al, Frailty measures, inflammatory biomarkers and post-operative complications in older surgical patients. Age Ageing. 2010;39:755758.

49. Qu, T., Walston, J.D., Yang, H. Upregulated ex vivo expression of stress-responsive inflammatory pathway genes by LPS-challenged CD14(+) monocytes in frail older adults. Mech Ageing Dev. 2009;130:161-166.

50. Qu, T., Yang, H., Walston, J.D. Upregulated monocytic expression of CXC chemokine ligand 10 (CXCL-10) and its relationship with serum interleukin-6 levels in the syndrome of frailty. Cytokine. 2009;46:319-324.

51. Fahey, J.L., Schnelle, J.F., Boscardin, J. Distinct categories of immunologic changes in frail elderly. Mech Ageing Dev. 2000;115:1-20.

52. Leng, S.X., Tian, X., Matteini, A. et al, Il-6-independent association of elevated serum neopterin levels with prevalent frailty in community-dwelling older adults. Age Ageing. 2011;40:475-481. 
53. Capuron, L., Schroecksnadel, S., Féart, C. et al, Chronic low-grade inflammation in elderly persons is associated with altered tryptophan and tyrosine metabolism: Role in neuropsychiatric symptoms. Biol Psychiatry. 2011;70:175-182.

54. Frick, B., Schroecksnadel, K., Neurauter, G. et al, Increasing production of homocysteine and neopterin and degradation of tryptophan with older age. Clin Biochem. 2004;37:684 687.

55. Kouchiwa, T., Wada, K., Uchiyama, M. et al, Age-related changes in serum amino acids concentrations in healthy individuals. Clin Chem Lab Med. 2012;50:861-870.

56. Pitkänen, H.T., Oja, S.S., Kemppainen, K. et al, Serum amino acid concentrations in aging men and women. Amino Acids. 2003;24:413-421.

57. Reibnegger, G., Huber, L.A., Jürgens, G. et al, Approach to define "normal aging" in man. Immune function, serum lipids, lipoproteins and neopterin levels. Mech Ageing Dev. 1988;46:67-82.

58. Spencer, M.E., Jain, A., Matteini, A. et al, Serum levels of the immune activation marker neopterin change with age and gender and are modified by race, BMI, and percentage of body fat. J Gerontol A Biol Sci Med Sci. 2010;65:858-865.

59. Ledochowski, M., Murr, C., Jäger, M., Fuchs, D. Dehydroepiandrosterone, ageing and immune activation. Exp Gerontol. 2001;36:1739-1747.

60. Pertovaara, M., Raitala, A., Lehtimäki, T. Indoleamine 2,3-dioxygenase activity in nonagenarians is markedly increased and predicts mortality. Mech Ageing Dev. 2006;127:497-499.

61. Solichova, D., Melichar, B., Blaha, V. Biochemical profile and survival in nonagenarians. Clin Biochem. 2001;134:563-569.

62. Theofylaktopoulou, D., Midttun Ulvik, A., Ueland, P.M. et al, A community-based study on determinants of circulating markers of cellular immune activation and kynurenines: The Hordaland Health Study. Clin Exp Immunol. 2013;173:121-130.

63. Vermeiren, S., Vella-Azzopardi, R., Beckwée, D. et al, Gerontopole Brussels study group. Frailty and the prediction of negative health outcomes: A meta-analysis. J Am Med Dir Assoc. 2016;17:1163.e1-1163.e17.

64. Chen, Y., Guillemin, G.J. Kynurenine pathway metabolites in humans: Disease and healthy states. Int J Tryptophan Res. 2009;2:1-19.

65. Taylor, M.W., Feng, G.S. Relationship between interferon-g, indoleamine 2,3dioxygenase, and tryptophan catabolism. FASEB J. 1991;5:2516-2522.

66. Capuron, L., Geisler, S., Kurz, K. et al, Activated immune system and inflammation in healthy ageing: Relevance for tryptophan and neopterin metabolism. Curr Pharm Des. 2014;20:6048-6057.

67. Sucher, R., Schroecksnadel, K., Weiss, G. et al, Neopterin, a prognostic marker in human malignancies. Cancer Lett. 2010;287:13-22.

68. Weinlich, G., Murr, C., Richardsen, L. et al, Decreased serum tryptophan concentration predicts poor prognosis in malignant melanoma patients. Dermatology. 2007;214:8-14.

69. Fuchs, D., Murr, C., Reibnegger, G. et al, Nitric oxide synthase and antimicrobial armature of human macrophages. J Infect Dis. 1994;169:224-225.

70. Scriver, C.R. The PAH gene phenylketonuria and a paradigm shift. Hum Mutat. 2007;28:831-845.

71. Mangge, H., Schnedl, W.J., Schrocksnadel, S. et al, Immune activation and inflammation in patients with cardiovascular disease are associated with elevated phenylalanine-totyrosine ratios. Pteridines. 2013;24:51-55.

72. Ploder, M., Neurauter, G., Spittler, A. et al, Serum phenylalanine in patients post trauma and with sepsis correlate to neopterin concentrations. Amino Acids. 2008;35:303-307.

73. Zangerle, R., Kurz, K., Neurauter, G. et al, Increased blood phenylalanine to tyrosine ratio in HIV-1 infection and correction following effective antiretroviral therapy. Brain Behav Immun. 2010;24:403-408.

74. Dorner, T.E., Luger, E., Tschinderle, J. et al, Association between nutritional status (MNA®-SF) and frailty (SHARE-FI) in acute hospitalised elderly patients. J Nutr Health Aging. 2014;18:264-269.

75. Pilleron, S., Ajana, S., Jutand, M.A. et al, Dietary patterns and 12-year risk of frailty: Results from the three-city Bordeaux study. J Am Med Dir Assoc. 2017;18:169-175.

76. Verlaan, S., Ligthart-Melis, G.C., Wijers, S.L.J. et al, High prevalence of physical frailty among community-dwelling malnourished older adults-A systematic review and metaanalysis. J Am Med Dir Assoc. 2017;18:374-382.

77. Nourhashémi, F., Andrieu, S., Gillette-Guyonnet, S. et al, Instrumental activities of daily living as a potential marker of frailty: A study of 7364 community-dwelling elderly women (the EPIDOS study). J Gerontol A Biol Sci Med Sci. 2001;56:M448-M453.

78. Chevalier, S., Saoud, F., Gray-Donald, K., Morais, J.A. The physical functional capacity of frail elderly persons undergoing ambulatory rehabilitation is related to their nutritional status. J Nutr Health Aging. 2008;12:721-726. 
79. Guigoz, Y., Doré, J., Schiffrin, J. The inflammatory status of old age can be nurtured from the intestinal environment. Curr Opin Clin Nutr Metab Care. 2008;11:13-20.

80. Biagi, E., Candela, M., Turroni, S. et al, Ageing and gut microbes: Perspectives for health maintenance and longevity. Pharmacol Res. 2013;69:11-20.

81. Cevenini, E., Monti, D., Franceschi, C. Inflamm-ageing. Curr Opin Clin Nutr Metab Care. 2013;16:14-20.

82. Claesson, M.J., Jeffery, I.B., Conde, S. et al, Gut microbiota composition correlates with diet and health in the elderly. Nature. 2012;488:178-184.

83. Jackson, M.A., Jeffery, I.B., Beaumont, M. et al, Signatures of early frailty in the gut microbiota. Genome Med. 2016;8:8.

84. van Tongeren, S.P., Slaets, J.P., Harmsen, H.J., Welling, G.W. Fecal microbiota composition and frailty. Appl Environ Microbiol. 2005;71:6438-6442. 